\title{
Screening, Management, and Acceptance of Patients with Aorto-Iliac Vascular Disease for Kidney Transplantation: A Survey among 161 Transplant Surgeons
}

\author{
Elsaline Rijkse $^{\mathrm{a}}$ Hendrikus J.A.N. Kimenai ${ }^{\mathrm{a}}$ Frank J.M.F. Dor ${ }^{\mathrm{b}, \mathrm{c}}$ \\ Jan N.M. IJzermans ${ }^{a}$ Robert C. Minnee ${ }^{a}$ \\ aDivision of HPB and Transplant Surgery, Department of Surgery, Erasmus MC Transplant Institute, Rotterdam, \\ The Netherlands; 'b Imperial College Transplant Centre, Hammersmith Hospital, Imperial College Healthcare NHS Trust, \\ London, UK; ' Department of Surgery and Cancer, Imperial College, London, UK
}

\section{Keywords \\ Kidney transplantation · Atherosclerosis · Recipient management}

\begin{abstract}
Introduction: Aorto-iliac vascular disease (AVD) is frequently found during the workup for kidney transplantation. However, recommendations on screening and management are lacking. We aimed to assess differences in screening, management, and acceptance of these patients for transplantation by performing a survey among transplant surgeons. Second, we aimed to identify center- and surgeon-related factors associated with decline or acceptance of kidney transplant candidates with AVD. Methods: A survey was sent to transplant surgeons and urologists. The survey contained general questions (part I) and 2 patient-based cases (part II) with Trans-Atlantic Inter-Society Consensus (TASC) D and B AVD supported with videos of their CT scans. Results: One hundred ninety-one (20.3\%) participants responded; 171 were currently involved in kidney transplantation: 161 (94.2\%) completed part I and 145 (84.8\%) part II. Screening for AVD was often (38.5\%) restricted to high-risk patients. The majority of respondents (67.7\%) rated "technical prob-
\end{abstract}

lems" as the most important concern in case of AVD, followed by "increased mortality risk because of cardiovascular comorbidity" (29.8\%). Pretransplant vascular interventions to facilitate transplantation were infrequently performed (71.4\% mentioned $<10$ per year). Ninety (64.3\%) respondents answered that an open vascular procedure should preferably be performed prior to kidney transplantation while $42(30.0 \%)$ respondents preferred a simultaneous open vascular procedure. The decline rate was higher in the TASC D case compared to the TASC B case $(26.9 \%$ and $9.7 \%$, respectively). Respondents from centers with expertise in pretransplant vascular interventions were more likely to accept both patients with TASC D and B for transplantation. Conclusion: There is no uniformity in the screening, management, and acceptance of patients with AVD for transplantation. If a center declines a patient with AVD because of technical concerns, the patient should be referred for a second opinion to a tertiary center with expertise in pretransplant vascular interventions. Multidisciplinary meetings including a vascular surgeon and a cardiologist could help optimize these patients for transplantation.

(C) 2021 The Author(s)

Published by S. Karger AG, Basel
(C) 2021 The Author(s)

Published by S. Karger AG, Basel

This is an Open Access article licensed under the Creative Commons Attribution-NonCommercial-4.0 International License (CC BY-NC) (http://www.karger.com/Services/OpenAccessLicense), applicable to the online version of the article only. Usage and distribution for commercial purposes requires written permission.
Correspondence to:

Elsaline Rijkse, a.rijkse@erasmusmc.nl 


\section{Introduction}

Aorto-iliac vascular disease (AVD) is frequently found during the workup for kidney transplantation due to increasing age and comorbidities accompanying end-stage renal disease [1]. The main concern in case of AVD is a technical failure of the vascular anastomosis, as the donor kidney is usually connected to the common or external iliac artery. Studies have shown that patients with AVD have a reduced life expectancy and an increased risk of cardiovascular events [2-4]. Severe calcification can cause a hemodynamically significant stenosis which needs repair in case its location is in the inflow tract of the kidney graft. However, also in case of nonstenotic AVD, an additional procedure may be needed to facilitate kidney transplantation if there is no soft artery left for the arterial anastomosis or clamping. Therefore, evaluation of vascular status and associated cardiovascular disease is of paramount importance in the kidney transplantation workup.

Notwithstanding the importance of this condition, clear recommendations on the management of these patients are lacking. The Kidney Disease Improving Global Outcomes (KDIGO) guidelines state that AVD is a relative contraindication for transplantation, but that selected patients can be considered for an endovascular or open vascular procedure to facilitate transplantation [5]. However, this guideline does not explain which patients are eligible for these procedures. Recommendations as whether to consider a patient eligible for transplant are also based on estimated life expectancy. However, minimum estimated life expectancy in relation to suitability for transplantation is often not quantified in guidelines [6]. As a result of this inconsistency, patients may be declined in acenter while they would have been accepted for transplantation elsewhere.

As a consequence of these variations in management and guidelines, patients with AVD may have inequitable access to transplantation. It is unknown which factors are associated with the decision whether to accept or decline a kidney transplant candidate with AVD. Therefore, the aim of the present study is 2 -fold: first, to assess differences in screening and management of these patients and second, to identify center- and surgeon-related factors associated with either decline or acceptance of kidney transplant candidates with AVD.

\section{Materials and Methods}

\section{Study Participants}

An online survey was conducted and sent to transplant surgeons, fellows, and urologists involved in kidney transplantation.
A list of eligible participants was compiled, consisting of participants from previous surveys that were carried out in our own center. This list was supplemented with email addresses of eligible participants received from the department heads of the Eurotransplant region and from participants of the Living Donor Nephrectomy course (endorsed by the European Society of Organ Transplantation) who had no objections to be contacted in the future. Respondents who were not involved in kidney transplantation could opt out of the survey.

\section{Survey Design}

The survey was conducted using Qualtrics ${ }^{\mathrm{XM}}$ software (Provo, UT, USA) and sent to all eligible participants between March and April 2019. Reminders were sent to nonrespondents 2 weeks after the initial invitation with a total of 3 reminders. The survey contained 2 parts: the first part contained general questions about the policy in the transplant center of the respondent and the second part contained 2 interactive cases. Two real-time cases with TransAtlantic Inter-Society Consensus (TASC) D and B were discussed together with a video of the contrast-enhanced CT scan and radiology report [7]. Consequently, respondents were asked if they considered the patient suitable for transplantation and if they would perform an additional vascular procedure. After the first decision, respondents were shown survival curves of transplanted patients with similar TASC AVD from the Erasmus MC University Medical Center and subsequently asked if this would change their decision. The 2 interactive cases including the complete survey and the survival curves of transplanted patients with similar AVD can be found in online supplementary Table 1 and online supplementary Figure S1 (for all online suppl. material, see www. karger.com/doi/10.1159/000519208).

\section{Statistical Analysis}

Continuous variables were presented as median with interquartile range (IQR) and compared with the Mann-Whitney U test or Kruskal Wallis test. Categorical variables were presented as number with percentage, and groups were compared with the $\chi^{2}$ test or Fisher's exact test. The McNemar test was used to compare paired categorical data and Wilcoxon signed rank test for paired continuous data. A complete case analysis was performed for the first and second part of the survey separately. In order to calculate odds ratio (OR) to identify factors associated with acceptance for transplant, a univariable logistic regression analysis was performed for each factor. A 2 -sided $p$ value $<0.05$ was considered significant. $\mathrm{R}$ statistical software (version 4.0.4) was used for all analyses, and GraphPad Prism (version 5.0) was used for data visualization.

\section{Results}

\section{Characteristics of the Respondents}

A total number of 939 potential kidney transplant surgeons were approached to participate in the online survey. Online supplementary Figure S2 shows the flowchart of included respondents, and Table 1 shows baseline characteristics of respondents who completed part I. Most respondents (60.9\%) were weekly involved in kid- 
Table 1. Baseline characteristics of the full respondents of part I

\begin{tabular}{|c|c|}
\hline & $\begin{array}{l}\text { Respondents, } \\
n(\%)\end{array}$ \\
\hline \multicolumn{2}{|l|}{ Frequency of KTx } \\
\hline Weekly & $98(60.9)$ \\
\hline Monthly & $47(29.2)$ \\
\hline Occasionally & $16(9.9)$ \\
\hline \multicolumn{2}{|c|}{ Continent of residency } \\
\hline Europe & $122(75.8)$ \\
\hline North America & $12(7.5)$ \\
\hline South America & $4(2.5)$ \\
\hline Africa & $3(1.9)$ \\
\hline Asia & $18(11.2)$ \\
\hline Oceania & $2(1.2)$ \\
\hline \multicolumn{2}{|c|}{ KTx per surgeon in their career, $N$} \\
\hline$<100$ & $27(16.8)$ \\
\hline $100-300$ & $55(34.2)$ \\
\hline $300-600$ & $43(26.7)$ \\
\hline$\geq 600$ & $36(22.4)$ \\
\hline \multicolumn{2}{|c|}{ Size of KTx program (per year) } \\
\hline$<100$ & $66(41.0)$ \\
\hline $100-200$ & $64(39.8)$ \\
\hline $200-300$ & $26(16.1)$ \\
\hline$\geq 300$ & $5(3.1)$ \\
\hline \multicolumn{2}{|c|}{ Experience with pretransplant vascular intervention } \\
\hline None & $21(13.0)$ \\
\hline$<10$ per year & $115(71.4)$ \\
\hline$\geq 10$ per year & $25(15.5)$ \\
\hline All respondents & 161 \\
\hline
\end{tabular}

ney transplantation, were from Europe (75.8\%), and had performed between 100 and 300 kidney transplantations in their career (34.2\%). They often worked in transplant centers where $<100$ kidney transplants per year were performed $(41.0 \%)$. The majority of respondents $(71.4 \%)$ worked in a center where $<10$ pretransplant vascular interventions were performed yearly.

\section{Part I: Policy regarding Vascular Screening and Management}

Most respondents (38.5\%) answered that only highrisk patients received vascular screening (Fig. 1a). A small proportion of respondents (14.9\%) answered that all adult patients received vascular screening while others (17.4\%) used an age cutoff with a median age of 50 years (IQR 40-50). Most respondents (53.4\%) employed a combination of multiple imaging modalities for vascular screening (Fig. 1b). In this case, a combination of duplex and CT scan was mentioned most frequently $(n=59$, $68.6 \%)$. The majority of respondents (71.4\%) answered

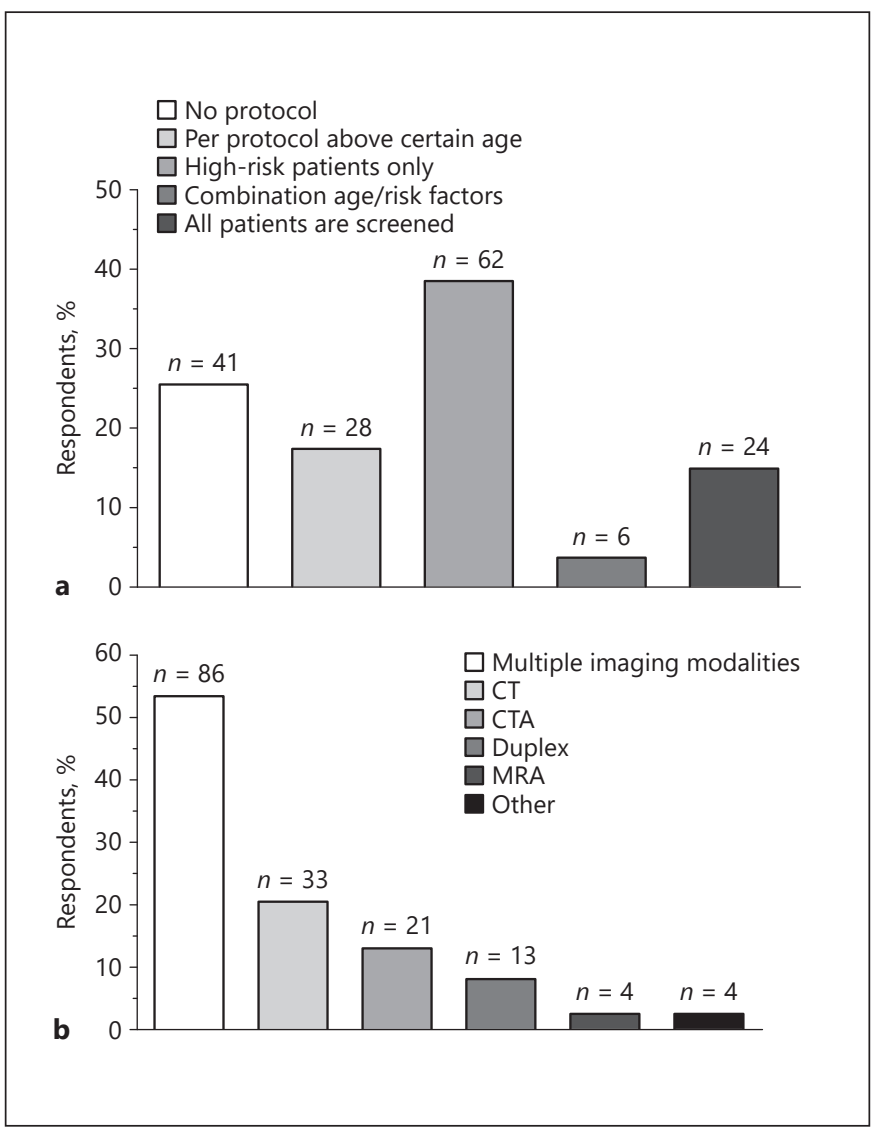

Fig. 1. a Vascular screening protocols currently in place. b Preferred type of imaging for vascular screening.

that vascular procedures to facilitate transplantation were performed $<10$ times per year (Fig. 2a). Most respondents who worked in hospitals with experience in these procedures $(87.0 \%)$ answered that an endovascular intervention should ideally take place prior to kidney transplantation (Fig. 2b). For open vascular procedures, 90 respondents (64.3\%) answered this should preferably be performed prior to kidney transplantation and 42 (30.0\%) simultaneously (Fig. 2c). From these 90 respondents, the majority (40.0\%) answered that there are no guidelines about the optimal length of time between vascular surgery and transplantation. Other respondents answered ideally $<6$ months (33.3\%), 6-12 months ( $25.6 \%)$, or longer than a year (1.1\%). Most respondents $(n=109,67.7 \%)$ rated "technical problems" as the most important concern, followed by "increased mortality risk because of cardiovascular comorbidity" ( $n=48,29.8 \%$ ) and "ethical concerns" ( $n=4,2.5 \%)$. According to the respondents, median minimal estimated life expectancy to be eligible for transplantation should be 10 years (IQR 5-12) in case 
of a living donor and 5 years (IQR 5-10) in case of a deceased donor ( $p$ value $<0.001$ ). An additional figure, stratifying minimal estimated life expectancy according to the respondent country, is shown in online supplementary Figure S3. This subgroup analysis was only performed if at least 10 respondents were available for a country. Considerable variation was observed in the minimal estimated life expectancy between respondent countries both in case of a deceased and living kidney transplant (overall $p$ value $<0.001$ and 0.007 , respectively).

\section{Part II: Interactive Cases}

Last, the respondents were asked how they would manage 2 patient cases with TASC D and B AVD. The percentage of patients that were deemed unsuitable for transplantation was higher in the TASC D case $(26.9 \%)$ compared to the TASC B case (9.7\%) (Fig. 3a). For the TASC D case, most respondents (35.2\%) would perform a vascular bypass/endarterectomy. For the TASC B case, most respondents (54.5\%) preferred percutaneous transluminal angioplasty and stenting (Fig. 3a). Table 2 shows the results from univariable logistic regression analysis to identify factors associated with acceptance for transplant compared to respondents who decided to decline the patient. In both the first and second case, respondents who accepted the patient were significantly more often working in a hospital with experience in pretransplant vascular interventions \{TASC D: OR 3.7 (95\% confidence interval [CI] 1.2-12.2) and TASC B: OR 16.3 (95\% CI: 4.5-62.4)\}. Other factors were not significantly associated with acceptance for transplant. After showing the survival curve, the acceptance rate decreased significantly in both cases with a stronger decline in the TASC D case (Fig. 3b, c, respectively).

\section{Discussion}

The first aim of the study was to assess differences in the screening and management of transplant candidates with AVD across different transplant centers. We found that there is no uniformity in the screening and management of these patients. Vascular screening is not only important to detect extensive calcifications that may complicate the transplant procedure but also to evaluate hemodynamically significant stenotic lesions as a potential source of inflow problems to the donor kidney. Most respondents used a combination of duplex and noncontrast-enhanced CT scan for vascular screening. The benefit of combining these 2 techniques is that both vascular calcification and vascular
How often is a vascular intervention in preparation for a kidney transplant performed in your center?

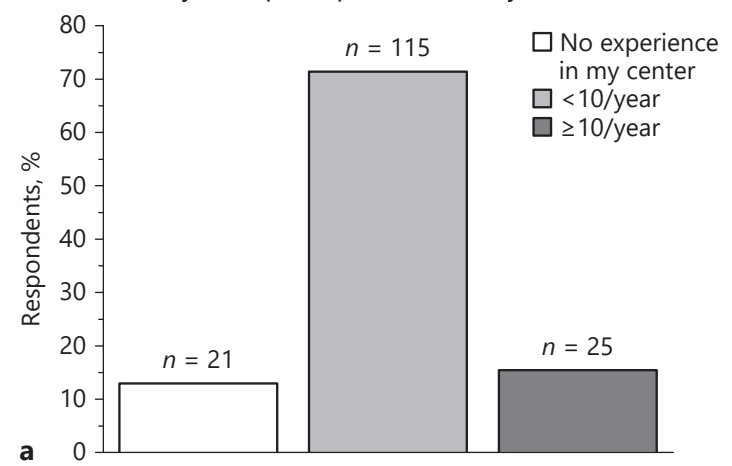

When should an endovascular intervention ideally take place?

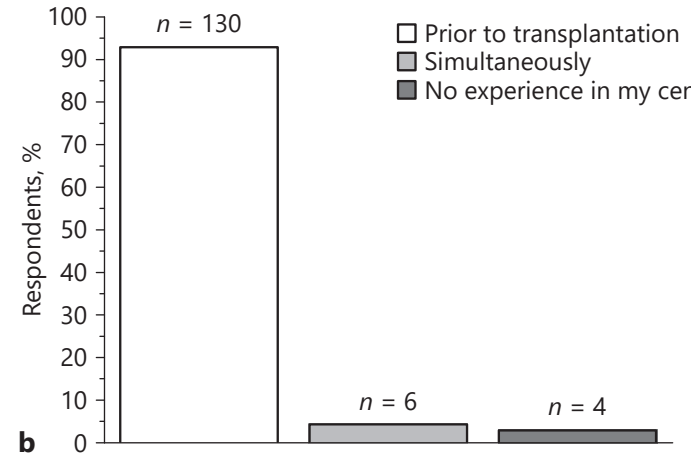

When should an open vascular intervention ideally take place?

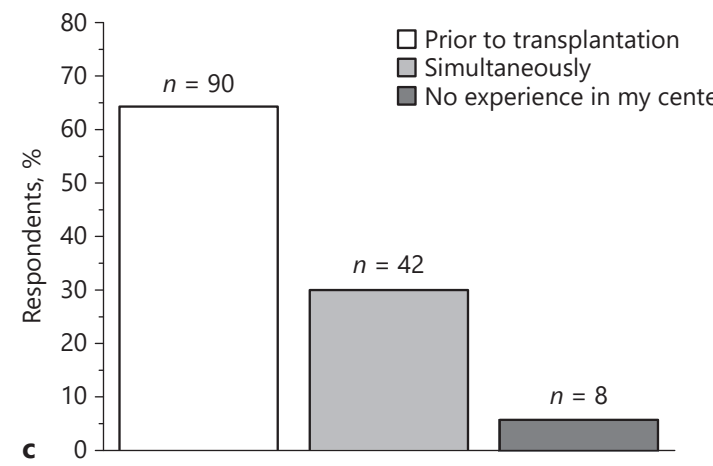

Fig. 2. Answers of the respondents to the questions whether vascular interventions are currently performed in preparation for transplantation (a), ideal timing of an endovascular intervention (b), and ideal timing of an open vascular intervention (c).

stenosis can be investigated without the risk of contrastinduced anaphylaxis or nephrotoxicity. As duplex is a noninvasive screening method with acceptable sensitivity (86\%) for identifying aorto-iliac stenosis, this may be a cost-effective first screening strategy $[8,9]$. 

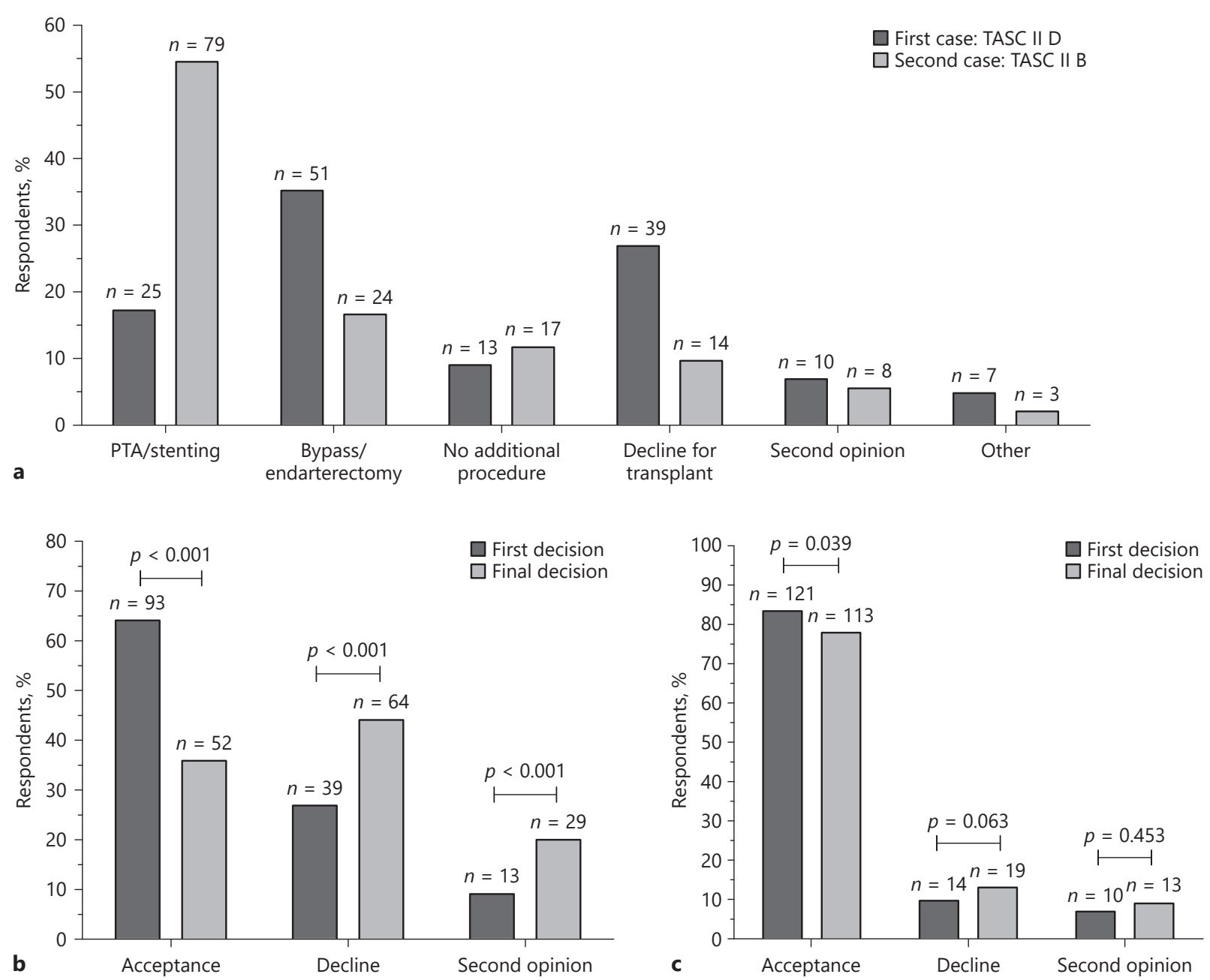

Fig. 3. a Opinion of the respondent on the management of the first case (TASC D) and second case (TASC B), based on contrast-enhanced imaging. The decision to decline or accept a patient or refer for a second opinion before and after showing the survival curve for the TASC D case (b) and the TASC B case (c). TASC, Trans-Atlantic Inter-Society Consensus.

Experience with pretransplant endovascular or open vascular interventions to facilitate transplantation is limited as most respondents worked in transplant centers where $<10$ of these procedures are yearly performed. Almost all respondents agreed that an endovascular intervention should ideally take place before transplantation. Endovascular interventions are generally recommended in TASC A and B AVD and have lower morbidity and mortality when compared to open vascular surgery $[10,11]$. In line with this, most respondents answered that an endovascular intervention was preferred in the TASC B case and an open procedure in the TASC D case. There is some de- bate whether lesions should be directly stented or to perform selective stenting if hemodynamic results are inadequate. Currently, stents are often selectively used, but there are also data to support the clinical efficacy of primary stent placement [12]. In the case of primary stent placement, the stent is placed regardless of the outcome of predilatation with percutaneous transluminal angioplasty. Especially in our kidney transplant candidates, selective stenting may be preferred, as stent placement may also complicate the renal artery anastomosis. Unfortunately, we did not ask this in our survey, which makes statements about the current practice on this topic not possible. 
Table 2. Univariable logistic regression analysis to identify associations between characteristics of the respondents and the odds of acceptance for transplant

\begin{tabular}{|c|c|c|c|c|c|c|}
\hline & \multicolumn{3}{|l|}{ First case: TASC D } & \multicolumn{3}{|l|}{ Second case: TASC B } \\
\hline Occasionally/monthly & $48(36.4)$ & Ref & \multirow{2}{*}{0.746} & 50 & Ref & \multirow{2}{*}{0.914} \\
\hline Weekly & $84(63.6)$ & $1.1(0.5-2.4)$ & & 85 & $0.9(0.3-2.9)$ & \\
\hline \multicolumn{7}{|l|}{ Continent } \\
\hline \multicolumn{7}{|c|}{ KTx per surgeon in their career, $N$} \\
\hline$<100$ & 18 (13.6) & Ref & \multirow{4}{*}{0.829} & 19 & Ref & \multirow{4}{*}{0.523} \\
\hline $100-300$ & $42(31.8)$ & $0.6(0.1-1.9)$ & & 44 & $2.6(0.4-15.2)$ & \\
\hline $300-600$ & $39(29.5)$ & $0.6(0.2-2.2)$ & & 39 & $2.3(0.4-13.4)$ & \\
\hline$\geq 600$ & $33(25.0)$ & $0.8(0.2-2.8)$ & & 33 & $1.1(1.2-4.9)$ & \\
\hline \multicolumn{7}{|c|}{ Size of KTx program (per year) } \\
\hline Yes & $118(89.4)$ & $3.7(1.2-12.2)$ & $0.023^{*}$ & 121 & $16.3(4.5-62.4)$ & $<0.001^{*}$ \\
\hline \multicolumn{7}{|l|}{ First concern } \\
\hline Technical & $87(65.9)$ & Ref & \multirow{3}{*}{0.737} & 89 & Ref & \multirow{3}{*}{0.161} \\
\hline Cardiovascular & $41(31.1)$ & $0.7(0.3-1.7)$ & & 42 & $0.4(0.1-1.2)$ & \\
\hline Ethical & $4(3.0)$ & $1.1(0.1-23.7)$ & & 4 & $0.2(0.0-4.7)$ & \\
\hline Total & 132 & & & 135 & & \\
\hline
\end{tabular}

$\mathrm{Cl}$, confidence interval; KTx, kidney transplantation; TASC, Trans-Atlantic Inter-Society Consensus. * Statistically significant.

There was no consensus about the optimal timing of an open vascular procedure. The literature is scarce on this topic, and only case series have been described [1320 ]. Benefits of a staged procedure are a lower risk of kidney thrombosis, a lower infection risk of the prosthesis, and stabilization of the prosthesis within surrounding tissue before transplantation [14]. However, a simultaneous procedure may be preferred in patients with a high operation risk due to cardiovascular comorbidities. Moreover, blood transfusions after open vascular surgery could lead to HLA sensitization, which may adversely affect access to transplantation in case of a staged procedure [21]. Therefore, the decision whether to do a staged or simultaneous procedure should be based on patient-specific comorbidities and current immunization status. Prediction tools to estimate the risk of cardiac complications after noncardiac surgery, such as the revised cardiac risk index, can be used to help decide whether to do a staged or simultaneous procedure [22].

Current guidelines consist of various recommendations with relative and absolute contraindications for kidney transplantation, aiming to balance the advantage of trans- plantation with the risks and the scarcity of donor organs [5]. The first reason why AVD is a relative contraindication is because of technical challenges, which may complicate a successful anastomosis. However, as previously mentioned, it has been shown that an endovascular or open vascular intervention can facilitate transplantation. One other reason may be that patients with AVD have a reduced life expectancy. In general, studies found that patients with comorbidities have reduced access to transplantation due to inferior survival [23]. We can confirm this with our survey for patients with AVD. Even though most respondents $(67.6 \%)$ answered that technical problems were the first concern when transplanting patients with $A V D$, we observed a drastic reduction in the willingness to accept the patient with TASC D for transplant after showing the survival curve to the respondents $(64.1 \%-35.9 \%)$. This indicates that inferior survival plays an important role in acceptance of these patients for transplant.

Compared to cutoff values for estimated life expectancy used in the literature, the respondents of the present study tended to be more conservative with a median minimal estimated life expectancy of at least 10 years for recipients 
of a living donor and 5 years for recipients of a deceased donor [6]. Consensus guidelines regarding minimal estimated life expectancy are important to assure similar standard of care and equal access to transplantation. However, between-country variation in deceased donation rates and geographical differences in life expectancy complicate applying international guidelines on this topic. We showed considerable between-country variation when the analysis of minimal estimated life expectancy was stratified according to the country of the respondent. Unfortunately, the low amount of respondents per country limits further analysis of these differences. One could hypothesize that the variation we observed is only due to between-country differences, and more uniformity is expected within countries. Still, a study has shown considerable disagreement in the acceptance of transplant candidates with relative contraindications between different transplant centers within the Netherlands [24]. This means that uniformity lacks on a national level, even in a small country as the Netherlands. We stretch the importance of national guidelines to provide similar access to transplantation for all patients with relative contraindications. This is in particular important in case of deceased donor kidney transplantation because of organ scarcity. In case of a living donor kidney transplantation, exclusion for transplant based on estimated life expectancy may not be justified because this does not affect the deceased donor pool [25].

Respondents from centers familiar with pretransplant vascular interventions to facilitate transplantation were more likely to accept these technically challenging patients in both the TASC D and B case. The odds ratio for acceptance in case the respondent came from an experienced center was much higher in the TASC B case (OR 16.3) compared to the TASC D case (OR 3.7). This may be because the first case with TASC D had the most severe $A V D$, resulting in also liberal respondents declining this patient for transplant. The decline in acceptance rate after the survival curve was larger in the TASC D case. This was as expected, as the survival curve of the TASC D case showed much inferior survival than the TASC B case. Our survey has several limitations. One limitation is nonresponse bias, which affects generalizability of the results. However, our study showed a moderate response rate, which was comparable to the expected average response rate of external group surveys. Our survey was distributed to a large group of transplant surgeons from all over the world. Differences in transplant programs and deceased donation rates make it difficult to make strong conclusions based on our data. We found large differences in the 2 cases whether respondents would decline or accept the patient for transplantation, which may also be largely distributed to geographical differences.

In conclusion, there is no uniformity in the screening, management, and acceptance of patients with AVD for transplantation. Considering important geographical differences, we suggest the need for national guidelines on the minimal estimated life expectancy to consider a patient with comorbidities eligible for transplant. If a center declines a patient with AVD because of technical concerns, the patient should be referred for a second opinion to a tertiary center with expertise in pretransplant vascular interventions. Multidisciplinary meetings, including a vascular surgeon and a cardiologist, could help optimize these patients before transplant to improve surgical success. Finally, more research is needed to investigate which patients benefit more from a staged or simultaneous open vascular procedure and who should be selected for an additional vascular procedure to facilitate transplantation. National registries should also collect data on the presence of AVD as an additional comorbidity and whether a staged or simultaneous additional vascular procedure was performed. This allows for larger cohort studies with sufficient power to study survival outcomes. Furthermore, this information may help answer the question which patients should be selected for a staged or simultaneous procedure. Including AVD as a variable in the dialysis and transplantation registry may also help identify which patients benefit from kidney transplantation in terms of perioperative mortality risk and survival.

\section{Acknowledgments}

We would like to thank all respondents for filling out the survey and providing us with useful feedback.

\section{Statement of Ethics}

The Medical Ethics Committee of the Erasmus Medical Center declared the study exempt from approval as the study is not subject to the Medical Research Involving Human Subjects Act (MEC 2019-373).

\section{Conflict of Interest Statement}

The authors have no conflicts of interest to declare.

\section{Funding Sources}

There were no sources of funding in relation to this work. 


\section{Author Contributions}

E.R., H.J.A.N.K., and R.C.M. contributed to conceptualization. E.R., H.J.A.N.K., F.J.M.F.D., and R.C.M. contributed to investigation. E.R. and R.C.M. contributed to formal analysis. E.R. and R.C.M. contributed to methodology. J.N.M.IJ. and R.C.M. contributed to supervision. E.R. contributed to writing - original draft. F.J.M.F.D. and J.N.M.IJ. contributed to writing - review and editing.

\section{Data Availability Statement}

The data of this study can be made available upon request.

\section{References}

1 Hernandez D, Rufino M, Bartolomei S, Gonzalez-Rinne A, Lorenzo V, Cobo $\mathrm{M}$, et al. Clinical impact of preexisting vascular calcifications on mortality after renal transplantation. Kidney Int. 2005;67(5):2015-20.

2 Rijkse E, van Dam JL, Roodnat JI, Kimenai HJAN, IJzermans JNM, Minnee RC. The prognosis of kidney transplant recipients with aorto-iliac calcification: a systematic review and meta-analysis. Transpl Int. 2020;33(5): 483-96.

3 Benjamens S, Pol RA, Glaudemans AWJM, Wieringa I, Berger SP, Bakker SJL, et al. A high abdominal aortic calcification score by dual X-ray absorptiometry is associated with cardiovascular events after kidney transplantation. Nephrol Dial Transplant. 2018;33(12): 2253-9.

4 Rijkse E, Kimenai HJAN, Roodnat JI, Ten Raa S, Bijdevaate DC, van Dam JL, et al. Impact of aortoiliac stenosis on graft and patient survival in kidney transplant recipients using the TASC II classification. Transplantation. 2019; 103(10):2164-72.

5 Chadban SJ, Ahn C, Axelrod DA, Foster BJ, Kasiske BL, Kher V, et al. KDIGO clinical practice guideline on the evaluation and management of candidates for kidney transplantation. Transplantation. 2020;104:S11-03.

6 Batabyal P, Chapman JR, Wong G, Craig JC, Tong A. Clinical practice guidelines on waitlisting for kidney transplantation: consistent and equitable? Transplantation. 2012;94(7): 703-13.

7 Norgren L, Hiatt WR, Dormandy JA, Nehler MR, Harris KA, Fowkes FG, et al. Inter-Society consensus for the management of peripheral arterial disease (TASC II). Eur J Vasc Endovasc Surg. 2007;33(Suppl 1):S1-75.

8 Koelemay MJ, den Hartog D, Prins MH, Kromhout JG, Legemate DA, Jacobs MJ. Diagnosis of arterial disease of the lower extremities with duplex ultrasonography. Br J Surg. 1996;83(3):404-9.
9 Visser K, de Vries SO, Kitslaar PJ, van Engelshoven JM, Hunink MG. Cost-effectiveness of diagnostic imaging work-up and treatment for patients with intermittent claudication in The Netherlands. Eur J Vasc Endovasc Surg. 2003;25(3):213-23.

10 Kashyap VS, Pavkov ML, Bena JF, Sarac TP, O'Hara PJ, Lyden SP, et al. The management of severe aortoiliac occlusive disease: endovascular therapy rivals open reconstruction. J Vasc Surg. 2008;48(6):1451-7.e1-3.

11 de Vries SO, Hunink MG. Results of aortic bifurcation grafts for aortoiliac occlusive disease: a meta-analysis. J Vasc Surg. 1997;26(4): 558-69.

12 Feldman DN, Armstrong EJ, Aronow HD, Banerjee S, Díaz-Sandoval LJ, Jaff MR, et al. SCAI guidelines on device selection in aortoiliac arterial interventions. Catheter Cardiovasc Interv. 2020;96(4):915-29.

13 Adamec M, Janouöek L, Toöenovský P, Lácha J. Renal transplantation combined with aortofemoral bypass using a fresh arterial allograft. Transpl Int. 2000;13(Suppl 1):S56-9.

14 Piquet P, Berland Y, Coulange C, Olmer M, Mercier C, Rampal M. Aortoiliac reconstruction and renal transplantation: staged or simultaneous. Ann Vasc Surg. 1989;3(3):2516.

15 Franquet Q, Terrier N, Pirvu A, Rambeaud JJ, Long JA, Janbon B, et al. Aortic bypass surgery for asymptomatic patients awaiting a kidney transplant: a word of caution. Clin Transplant. 2018;32(4):e13218.

16 Ozcelik A, Treckmann J, Paul A, Witzke O, Sotiropoulos G, Nadalin S, et al. Results of kidney transplantation with simultaneous implantation of vascular graft. Transplant Proc. 2007;39(2):509-10.
17 Patrono D, Verhelst R, Buemi A, Goffette P, De Pauw L, Kanaan N, et al. Renal allograft implantation on prosthetic vascular grafts: short- and long-term results. World J Surg. 2013;37(7):1727-34.

18 Sagban TA, Regus S, Heller K, Jacobi J, Apel $\mathrm{H}$, Keck B, et al. Results of renal transplantation on alloplastic arterial grafts. Urol Int. 2016;96(2):157-63.

19 Tozzi M, Franchin M, Soldini G, Ietto G, Chiappa C, Molteni B, et al. Treatment of aortoiliac occlusive or dilatative disease concomitant with kidney transplantation: how and when? Int J Surg. 2013;11(Suppl 1):S115-9.

20 Tsivian M, Neri F, Nardo B, Bertelli R, Cavallari G, Fuga G, et al. Aortoiliac surgery concomitant with kidney transplantation: a single center experience. Clin Transplant. 2009; 23(2):164-7.

21 Leffell MS, Kim D, Vega RM, Zachary AA, Petersen J, Hart JM, et al. Red blood cell transfusions and the risk of allosensitization in patients awaiting primary kidney transplantation. Transplantation. 2014;97(5):525-33.

22 Boersma E, Kertai MD, Schouten O, Bax JJ, Noordzij P, Steyerberg EW, et al. Perioperative cardiovascular mortality in noncardiac surgery: validation of the lee cardiac risk index. Am J Med. 2005;118(10):1134-41.

23 Segev DL, Kucirka LM, Oberai PC, Parekh RS, Boulware LE, Powe NR, et al. Age and comorbidities are effect modifiers of gender disparities in renal transplantation. J Am Soc Nephrol. 2009;20(3):621-8.

24 Glijn NH, Roodnat JI, Dor FJ, Betjes MG, Zuidema WC, Weimar W, et al. Kidney transplantation in patients declined by other centres. Neth J Med. 2017;75(2):74-80.

25 Wong G, Howard K, Chapman JR, Chadban $\mathrm{S}$, Cross N, Tong A, et al. Comparative survival and economic benefits of deceased donor kidney transplantation and dialysis in people with varying ages and co-morbidities. PLoS One. 2012;7(1):e29591. 